\title{
Generation of induced pluripotent mouse stem cells in an indirect co-culture system
}

\author{
F.L. Dong1,3, H.A. Kaleri' ${ }^{2}$, Y.D. Lu ${ }^{1}$, C.L. Song ${ }^{1}$, B.C. Jiang ${ }^{1}$, B.L. Zhang ${ }^{1}$, \\ L.J. Wang ${ }^{1}$, X.G. Wang ${ }^{1}$, X.S. Ma ${ }^{1}$, B.J. Wu ${ }^{4}$, H. Song ${ }^{5}$, J. Li ${ }^{1}$ and H.L. Liu ${ }^{1}$ \\ ${ }^{1}$ Department of Animal Breeding and Genetics, \\ College of Animal Science and Technology, Nanjing Agricultural University, \\ Nanjing, China \\ ${ }^{2}$ Department of Animal Breeding and Genetics, \\ Faculty of Animal Husbandry and Veterinary Sciences, \\ Sindh Agriculture University, Tando Jam, Pakistan \\ ${ }^{3}$ Institutes of Biology and Medical Sciences, Soochow University, \\ Suzhou, China \\ ${ }^{4}$ Inner Mongolia Saikexing Reproductive Biotechnology Co. Ltd., \\ Hohhot, China \\ ${ }^{5}$ Center of Animal Embryo Engineering and Technology, \\ Nanjing Agricultural University, Nanjing, China \\ Corresponding author: H.L. Liu \\ E-mail: liuhonglin@263.net
}

Genet. Mol. Res. 11 (4): 4179-4186 (2012)

Received November 30, 2011

Accepted August 15, 2012

Published December 6, 2012

DOI http://dx.doi.org/10.4238/2012.December.6.1

\begin{abstract}
Typically, production of induced pluripotent stem cells requires direct contact with feeder cells. However, once the stem cells have reached the appropriate maturation point, it is difficult to separate them from feeder cells, which must be irradiated with $\gamma$-rays or treated with the antibiotic mitomycin-C. We used a microporous poly-membrane-based indirect contact co-culture system with mouse embryonic fibroblasts to induce mouse pluripotent stem cells without radiation or antibiotics. We found that induced pluripotent stem cells induced by this co-culture method had a reprogramming efficiency and time similar to those induced using traditional methods. Furthermore,
\end{abstract}


strongly expressed pluripotent markers showed a normal karyotype and formation and contained all three germ layers in a teratoma.

Key words: Induced pluripotent stem cells; Feeder cells; Co-culture; Microporous polymer membrane

\section{INTRODUCTION}

Induced pluripotent stem cells (iPSCs) can be generated from somatic cells by the transduction of the transcription factors Oct3/4, Sox2, c-Myc, and Klf4 to reprogram them to an equivalent of the early embryonic state (Takahashi and Yamanaka, 2006; Okita et al., 2007). This discovery makes it possible to generate patient-specific stem cells and has the potential for relevant applications in regenerative medicine (Hanna et al., 2007). However, the constraints of this technology include low efficiency and long induction time (Maherali et al., 2008). To resolve these problems, the genetic mechanism of the induction process has become a focus of research. In previous studies, iPSCs were generated directly on feeder cells (Takahashi and Yamanaka, 2006; Okita et al., 2007). However, this method is not conducive to the sampling and removal of feeder cells during induction. The derivation of feeder-free iPSCs has been reported (Sun et al., 2009). However, feeder cells can produce multiple growth factors and some as yet unknown factors. In contrast, feeder-free systems do not provide all of these growth factors, which better stimulates the growth of stem cells in an in vitro environment (Lim and Bodnar, 2002; Soh et al., 2007; Eiselleova et al., 2008).

Herein, we report another method for the generation of mouse iPSCs. In this system, the cells to be induced do not contact feeder cells directly, but feeder cells can provide all of the growth factors necessary to maximize growth. In this co-culture system, mouse embryonic fibroblast (MEF) cells and iPSCs were physically separated by a microporous polyethylene terephthalate membrane before induction (Figure 1B). This co-culture system exhibited an induction efficiency similar to that of iPSC generation on feeder cells. Co-culture-iPSCs (CiPSCs) have the same characteristics, differentiation, expression of pluripotent markers, and morphology as embryonic stem cells (ESCs) grown in colonies in vitro. A previous study found that human ESCs and iPSCs can be propagated using this method (Kim et al., 2007; Abraham et al., 2010). In our study, we provide evidence that this culture system is convenient and simple to use in research concerning the reprogramming mechanism of stem cells in an in vitro environment. We found that cells induced with this method exhibit the same pluripotency in vivo.

\section{MATERIAL AND METHODS}

\section{Cell culture}

MEFs were derived from e13.5 embryos of C57BL6 mice and were maintained in Dulbecco's modified Eagle's medium (DMEM; high glucose), 10\% fetal bovine serum, LGlutaMax, non-essential amino acids (NEAA), sodium pyruvate, and penicillin/streptomycin. ESCs and iPSCs were cultivated on $\gamma$-irradiated MEFs in KnockOut ${ }^{\mathrm{TM}}$ DMEM, $20 \%$ KnockOut Serum Replacement (KSR), L-GlutaMax, NEAA, sodium pyruvate, penicillin/streptomycin, $0.1 \mathrm{mM} \beta$-mercaptoethanol, and $1000 \mathrm{U} / \mathrm{mL}$ leukemia inhibitory factor (Millipore, 
Billerica, MA, USA). Unless otherwise indicated, all reagents were purchased from Invitrogen Corporation (Carlsbad, CA, USA).

\section{Retrovirus production and generation of induced pluripotent stem cells}

PMX-based retroviral vectors (Addgene, Cambridge, MA, USA) containing the murine cDNAs of Oct4, Sox2, and Klf4 were introduced into platE cells using Lipofectamine 2000 (Invitrogen Corporation). MEFs within three passages were seeded at a density of 3500$5000 \mathrm{cells} / \mathrm{cm}^{2}$. Twelve hours later, MEFs were transduced with viral supernatants containing equal parts of the three transcription factors (Oct4, Sox2, Klf4; Takahashi et al., 2007). The cells were cultured initially in MEF medium. After infection, the medium was gradually replaced by ESC medium supplemented with $50 \mu \mathrm{g} / \mathrm{mL}$ vitamin C (Sigma-Aldrich Corporation, St. Louis, MO, USA) using a 3-day stepwise process to enhance induction efficiency (Chen et al., 2010; Esteban et al., 2010).

For direct feeder cell-induced conditions, the MEFs were induced on feeder cells; for the indirect co-culture system (Figure 1B), MEFs were induced on Millicell hanging inserts $(1.0-\mu \mathrm{m}$ polyethylene terephthalate membrane; Esteban et al., 2010), and MEFs were used as feeder cells.

\section{Immunofluorescence and alkaline phosphatase staining}

Cells were prepared for immunofluorescence after fixation in a 3.7\% paraformaldehyde solution at room temperature for $1 \mathrm{~h}$. They were then washed three times in phosphatebuffered saline (PBS) with $0.2 \%$ Tween-20 and left in blocking buffer (PBS/0.2\% Tween-20 and $10 \mathrm{mg} / \mathrm{mL}$ bovine serum albumin) at $37^{\circ} \mathrm{C}$ for $1 \mathrm{~h}$. Cells were then incubated in primary antibodies against Nanog (Abcam, Cambridge, MA, USA), SSEA-1 (Chemicon International Inc., Billerica, MA, USA), and OCT4 (Abcam) diluted 1:200-1:100 at $37^{\circ} \mathrm{C}$ for $1 \mathrm{~h}$ or overnight at $4{ }^{\circ} \mathrm{C}$. Cells were then washed three times in PBS $/ 0.2 \%$ Tween-20 and incubated with $\mathrm{Cy} 3$ - or fluorescein isothiocyanate-conjugated anti-mouse or anti-rabbit immunoglobulin $\mathrm{G}$ (IgG) secondary antibody $\left(1: 200\right.$; Abcam) at $37^{\circ} \mathrm{C}$ for $1 \mathrm{~h}$. Finally, cells were washed three times in PBS/0.2\% Tween-20, stained for DNA with $3 \mathrm{~g} / \mathrm{L}$ 4',6-diamidino-2-phenylindole for $20 \mathrm{~min}$, and were analyzed with a confocal immunofluorescence microscope. Alkaline phosphatase (AP) staining was performed using the Alkaline Phosphatase Detection kit (Millipore), following manufacturer instructions.

\section{RNA extraction and reverse-transcription polymerase chain reaction (RT-PCR)}

Total RNA was isolated using the Trizol reagent (Invitrogen Corporation) according to the manufacturer protocol. RNA integrity was verified by gel electrophoresis and ethidium bromide staining, and its purity was examined by ultraviolet spectrophotometry $(260 / 280 \mathrm{~nm}$ optical density). Two micrograms of total RNA was used to synthesize first-strand cDNA using the M-MLV reverse transcriptase (Promega, Madison, WI, USA) and oligo dT(18). Conventional PCRs were performed with the primer sequences shown in Table 1 (Chen et al., 2009, 2010). The expression levels of Nanog and SSEA-1 were assessed using FastStart Universal SYBR Green Master (ROX; Roche Diagnostics, Rotkreuz, Switzerland) and analyzed with an ABI7300 fluorescence quantitative PCR instrument. 
Table 1. Primers used in this study (Chen et al., 2009, 2010).

\begin{tabular}{lll}
\hline Gene name & Forward primer (5'-3') & Reverse primer (5'-3') \\
\hline Dax1 & GCTGGAGTCTGAACATTGAC & CAGTATGGAGCAGAGGGAT \\
Fbx15 & CTTACTTGGATGCGGTGAC & ATTCTGAGGGACTCTTTGG \\
Dppa5 & AACAACAAGATTCGGGCTAA & AAACTGGCTTCACTCGATACA \\
FGF4 & GTGGTGAGCATCTTCGGAGTGG & GCGTAGGATTCGTAGGCGTTGT \\
Nac1 & CCACAATGAAGAGGACGAAG & TTGCCAATCTGGTTGATAAGT \\
Oct4 & TAGGTGAGCCGTCTTTCCAC & TTCAGCCAGGTTCGAGGAT \\
Klf4 & AACATGCCCGGACTTACAAA & TGAAACCTGTCCTGGTCTTC \\
Ranog & CTCAAGTCCTGAGGCTGACA & GTCTCCGATTTGCATATCTCCTG \\
GAPDH & CAGCCAGACCACCATCTGTC & GTTGAAGTCGCAGGAGACAACC \\
\hline
\end{tabular}

\section{Teratoma formation and karyotype analysis}

To evaluate the pluripotency of C-iPSCs, 5 million cells were injected subcutaneously into SCID mice. Four weeks after injection, teratomas were collected and analyzed by hematoxylin and eosin staining following standard procedures. Karyotype analysis was performed as described previously (Esteban et al., 2009).

\section{RESULTS AND DISCUSSION}

\section{Generation of induced pluripotent stem cell colonies using the traditional method or a co-culture system}

Typically, murine iPSCs can be derived by direct contact with a feeder-cell layer. This direct pattern presents an obstacle for later separation of the feeder cells from the iPSCs during the middle stages of reprogramming. In this study, MEFs were used to generate iPSCs using three viral vectors with Vc (for timeline, see Figure 1A), either in a co-culture system (Figure 1B) or with direct contact of feeder cells. Similar to the traditional method, we observed colonies with an ESC-like morphology at 14 days post-infection that were enough big to select.

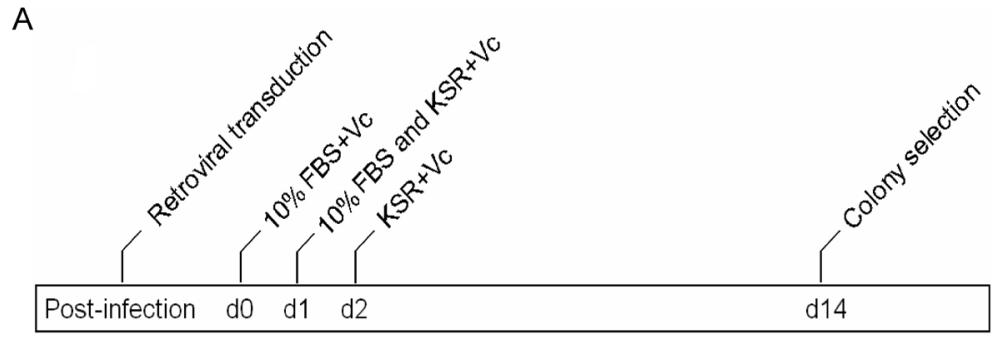

B

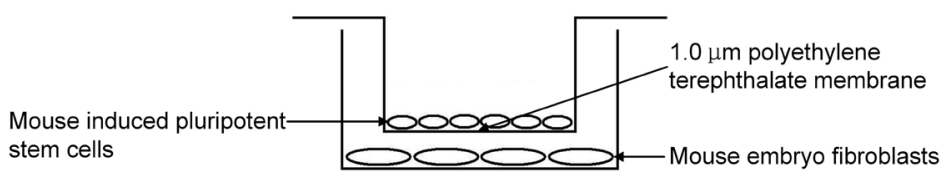

Figure 1. Schematic diagram. A. The timeline of induction of induced pluripotent stem cells by gradual replacement of the medium. B. Schematic diagram of co-culture system. FBS = fetal bovine serum; KSR = KnockOut Serum Replacement; $\mathrm{Vc}=$ vitamin C. 
These cells were located directly on feeder cells (Figure 2a) and in the indirect coculture system (Figure 2b). On the same day, these ESC-like colonies were stained with AP, and a positive result was observed for both methods (Figure $2 \mathrm{c}$ and d). Fourteen days after retroviral infection, we counted the number of AP-positive colonies (Figure 2g). By analyzing the data, we found that direct contact with feeder cells did not obviously increase the iPSC colony numbers or shorten the time of induction. C-iPSCs displayed a $0.205 \%$ reprogramming efficiency, and those derived using the traditional method (T-iPSCs) displayed a $0.19 \%$ reprogramming efficiency when repeated twice. Thus, the generation of iPSCs using a co-culture system did not reduce the reprogramming time or extend the time of induction. After colony selection, C-iPSCs were cultured directly on feeder cells over 10 generations and were observed to maintain a morphology similar to that of T-iPSCs (Figure 2e and f).
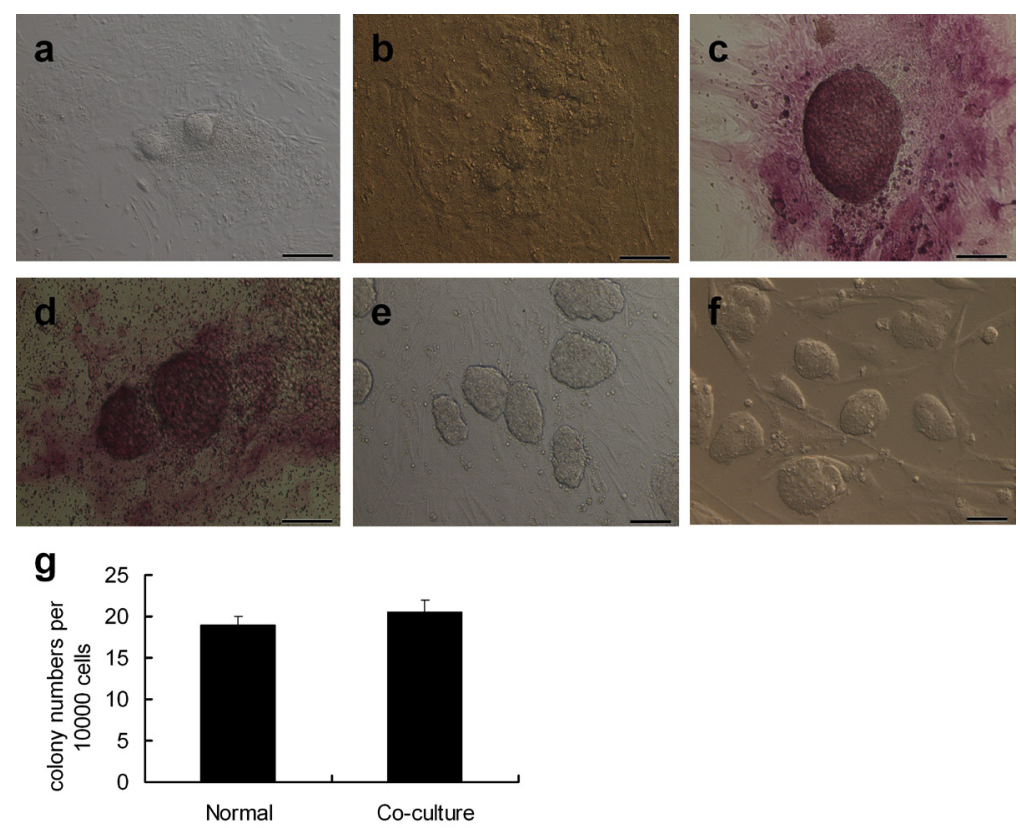

Figure 2. Comparison of the traditional method and co-culture-induced pluripotent stem cells. (a-d) Morphology. (c and d) Alkaline phosphatase (AP) staining. (a and b) Fourteen days after retroviral infection. (e and f) Morphology of 10 generations cultured directly on feeder cells after colony selection. (g) Comparison of the number of APpositive colonies per 10,000 cells at 14 days after retroviral infection using the traditional method and the co-culture system. (a, c and e) Inductions using the traditional method. (b, d and f) Induction using the co-culture system. (a, b, e, and f) Scale bars indicate $100 \mu \mathrm{m}$. (c and d) Scale bars indicate $200 \mu \mathrm{m}$.

\section{Embryonic stem cell markers expressed in co-culture-induced pluripotent stem cells}

To confirm that C-iPSCs can display characteristics similar to ESCs, we stained CiPSCs with SSEA-1, OCT4, and NANOG antibodies. The results indicated that these cells are positive for typical mESC markers (Figure 3A). RT-PCR showed that the cells expressed various mESC marker genes, including Oct3/4, Nanog, Klf4, Rex-1, Fbx15, Dppa5, Dax1, FGF4, and Nac1, at levels similar to those in mESCs (Figure 3B). Furthermore, to evaluate the 
relative gene expression of NANOG and REX-1, RNA from C-iPSCs and mESCs was isolated and analyzed by quantitative RT-PCR. Expression of NANOG and REX-1 was assessed using the $2^{-\Delta \Delta \mathrm{Ct}}$ method for each sample (Figure $3 \mathrm{C}$ ). The results showed that the expression levels of pluripotent genes were similar to those of mESCs.

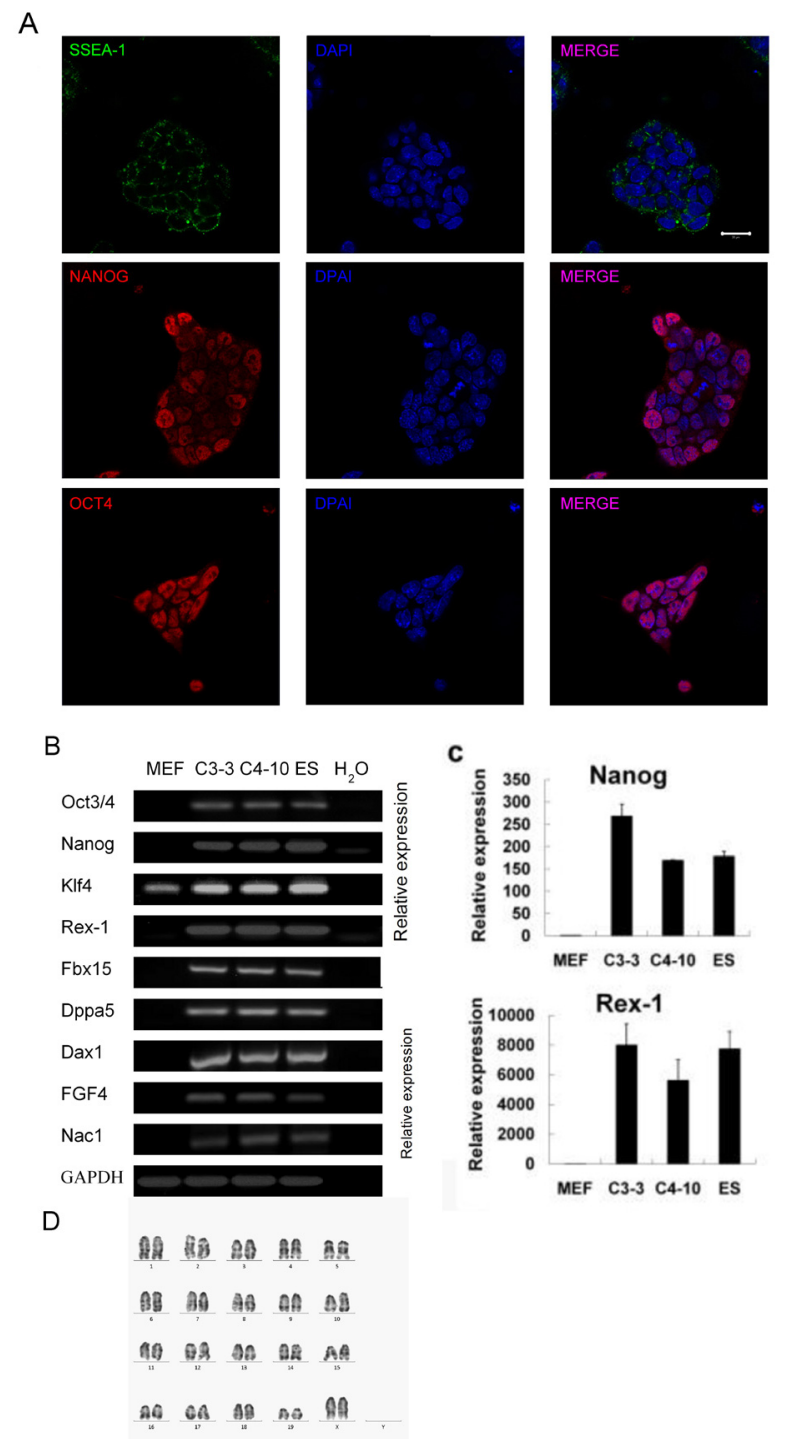

Figure 3. Embryonic stem (ES) cell markers expressed in co-culture-induced pluripotent stem cells (C-iPSCs). A. Immunofluorescence staining shows that C-iPSCs express ES cell marker genes, such as SSEA-1, Oct4, and Nanog. Scale bars indicate $20 \mu \mathrm{m}$. B. Reverse-transcription polymerase chain reaction analysis of ES cell marker genes in C-iPSCs for expression of Oct3/4, Nanog, Klf4, Rex-1, Fbx15, Dppa5, Dax1, FGF4, Nac1, and GAPDH. C. Expression levels of Nanog and Rex-1 in mouse embryonic fibroblasts (MEFs), ES cells, and C-iPSCs relative to GAPDH as assessed by quantitative PCR. Values from MEFs were set to 1. C3-3 and C4-10 are two C-iPSC lines. D. C-iPSCs displayed a normal karyotype. 


\section{Co-culture induced pluripotent stem cells maintained differentiation potential in vivo}

To test pluripotency in vivo, we injected C-iPSCs into nude mice to determine whether these cells produced teratomas. Thirty days after injection, teratomas were stained with hematoxylin and eosin, which revealed differentiated tissues in all three germ layers (Figure 4). Lastly, C-iPSCs displayed a normal karyotype (Figure 3D) and were maintained directly on feeder cells for more than 10 generations. Taken together, these results demonstrate that $\mathrm{C}$ iPSCs displayed characteristics similar to those of mESCs, with regard to both marker expression and differentiation potential in vivo.
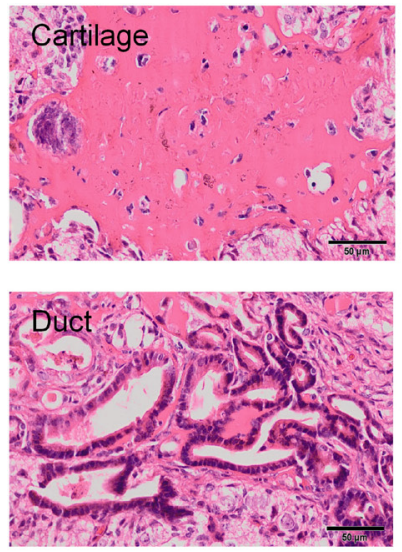
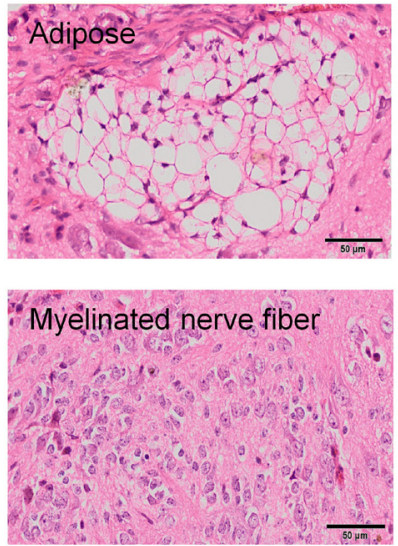
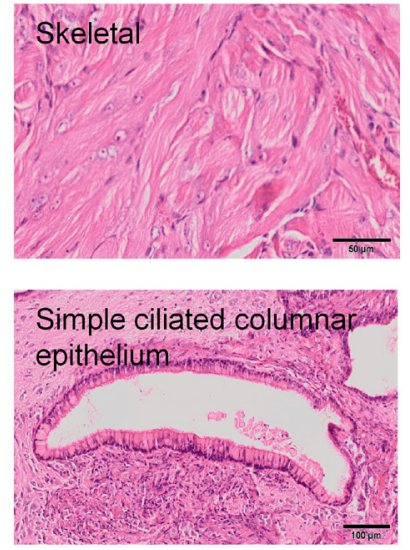

Figure 4. Hematoxylin and eosin staining of teratoma tissues from all three germ layers.

\section{CONCLUSIONS}

Generally, mitomycin-C-inactivated or $\gamma$-irradiated MEF cells are used for feeder cells to generate iPSCs, and feeder cells are in direct contact with the cells to be induced. Here, we describe a cell culture system that successfully generates mouse iPSCs without direct contact of feeder cells. Compared with traditional methods, this method has a similar reprogramming efficiency and time to maturation. C-iPSCs strongly expressed pluripotent markers and showed a normal karyotype. Furthermore, C-iPSCs had the ability to form teratomas after injection into nude mice, and three germ layers formed within 30 days. This method conveniently allows for the easy separation of feeder cells without enzyme treatment from iPSCs midway through the process of induction (Kim et al., 2007). In addition, MEF cells can be used for feeder cells directly.

\section{ACKNOWLEDGMENTS}

Research supported by the Key Project of Chinese National Programs for Fundamental Research and Development (973 Program \#2007CB947403). We are grateful to Fangxiong Shi for supplying the microtome and technical discussions. 


\section{REFERENCES}

Abraham S, Sheridan SD, Laurent LC, Albert K, et al. (2010). Propagation of human embryonic and induced pluripotent stem cells in an indirect co-culture system. Biochem. Biophys. Res. Commun. 393: 211-216.

Chen J, Liu J, Han Q, Qin D, et al. (2010). Towards an optimized culture medium for the generation of mouse induced pluripotent stem cells. J. Biol. Chem. 285: 31066-31072.

Chen M, Sun X, Jiang R, Shen W, et al. (2009). Role of MEF feeder cells in direct reprogramming of mousetail-tip fibroblasts. Cell Biol. Int. 33: 1268-1273.

Eiselleova L, Peterkova I, Neradil J, Slaninova I, et al. (2008). Comparative study of mouse and human feeder cells for human embryonic stem cells. Int. J. Dev. Biol. 52: 353-363.

Esteban MA, Xu J, Yang J, Peng M, et al. (2009). Generation of induced pluripotent stem cell lines from Tibetan miniature pig. J. Biol. Chem. 284: 17634-17640.

Esteban MA, Wang T, Qin B, Yang J, et al. (2010). Vitamin C enhances the generation of mouse and human induced pluripotent stem cells. Cell Stem. Cell 6: 71-79.

Hanna J, Wernig M, Markoulaki S, Sun CW, et al. (2007). Treatment of sickle cell anemia mouse model with iPS cells generated from autologous skin. Science 318: 1920-1923.

Kim S, Ahn SE, Lee JH, Lim DS, et al. (2007). A novel culture technique for human embryonic stem cells using porous membranes. Stem. Cells 25: 2601-2609.

Lim JW and Bodnar A (2002). Proteome analysis of conditioned medium from mouse embryonic fibroblast feeder layers which support the growth of human embryonic stem cells. Proteomics 2: 1187-1203.

Maherali N, Ahfeldt T, Rigamonti A, Utikal J, et al. (2008). A high-efficiency system for the generation and study of human induced pluripotent stem cells. Cell Stem. Cell 3: 340-345.

Okita K, Ichisaka T and Yamanaka S (2007). Generation of germline-competent induced pluripotent stem cells. Nature 448: 313-317.

Soh BS, Song CM, Vallier L, Li P, et al. (2007). Pleiotrophin enhances clonal growth and long-term expansion of human embryonic stem cells. Stem. Cells 25: 3029-3037.

Sun N, Panetta NJ, Gupta DM, Wilson KD, et al. (2009). Feeder-free derivation of induced pluripotent stem cells from adult human adipose stem cells. Proc. Natl. Acad. Sci. U. S. A. 106: 15720-15725.

Takahashi K and Yamanaka S (2006). Induction of pluripotent stem cells from mouse embryonic and adult fibroblast cultures by defined factors. Cell 126: 663-676.

Takahashi K, Okita K, Nakagawa M and Yamanaka S (2007). Induction of pluripotent stem cells from fibroblast cultures. Nat. Protoc. 2: 3081-3089. 